\title{
Generalized Jordan Sets in the Theory of Singular Partial Differential-Operator Equations $^{\star}$
}

\author{
Michael V. Falaleev, Olga A. Romanova, and Nicholas A. Sidorov \\ Irkutsk State University, 664003 Irkutsk, Russia \\ mihail@ic.isu.ru, olga@baikal.ru, sidorov@home.isu.runnet.ru
}

\begin{abstract}
We apply the generalized Jordan sets techniques to reduce partial differential-operator equations with the Fredholm operator in the main expression to regular problems. In addition this techniques has been exploited to prove a theorem of existence and uniqueness of a singular initial problem, as well as to construct the left and right regularizators of singular operators in Banach spaces and to construct fundamental operators in the theory of generalized solutions of singular equations.
\end{abstract}

\section{Introduction}

Let

$$
\begin{gathered}
x=\left(t, x^{\prime}\right) \text { be a point in the space } R^{m+1}, \\
x^{\prime}=\left(x_{1}, \ldots, x_{m}\right), \quad D=\left(D_{t}, D_{x_{1}}, \ldots, D_{x_{m}}\right), \\
\alpha=\left(\alpha_{0}, \ldots, \alpha_{m}\right), \quad|\alpha|=\alpha_{0}+\alpha_{1}+\cdots \alpha_{m}, \quad \text { where } \\
\alpha_{i} \text { are integer non-negative indexes, } D^{\alpha}=\frac{\partial^{\alpha}}{\partial t^{\alpha_{0}} \ldots \partial x_{m}^{\alpha_{m}}} .
\end{gathered}
$$

We also suppose that $B_{\alpha}: D_{\alpha} \subset E_{1} \rightarrow E_{2}$ are closed linear operators with dense domains in $E_{1}, x \in \Omega$, where $\Omega \subset R^{m+1},|t| \leq T, E_{1}, E_{2}$ are Banach spaces.

Let us consider the following operator

$$
L(D)=\sum_{|\alpha| \leq l} B_{\alpha} D^{\alpha} .
$$

The operator $\sum_{|\alpha|=l} B_{\alpha} D^{\alpha}$ we call the main part of $L(D)$. Due to its theoretical significance and numerous applications (see [1], 2], 8], 9]) the most interesting case is when the operator $L(D)$ contains a Fredholm operator $B_{l 0 \ldots 0} \equiv B$ in the higher derivative $D_{t}^{l}$.

If $N(B) \neq\{0\}$, then the operator $L(D)$ is called a singular differential operator. A singular operator of the first order

$$
B \frac{d}{d t}+A
$$

with the Fredholm operator $B$ when $x=t \in R^{1}$, has been investigated intensively. The significant results of these investigations were obtained due to new

\footnotetext{
* Supported in part by INTAS (grant No.2000-15).
} 
research approaches in the semigroup theory (the bibliography and mechanical models are represented in 9] ). The investigations similar to our research, considering the case when $x \in R^{m+1}$ are represented in [7, [8]. Below we use some results from [3], 4], 7].

We consider the equation

$$
L(D) u=f(x)
$$

where $f: \Omega \rightarrow E_{2}$ is an analytical function of $x^{\prime}$ sufficiently smooth of $\mathrm{t}$. The Cauchy problem for (3), when $E_{1}=E_{2}=R^{n}$ and the matrix $B=B_{l 0 \ldots 0}$ is not degenerated, has been thoroughly studied in fundamental papers by I.G. Petrovsky (see [5]). In the case when the operator $B$ is not invertible the theory of initial and boundary value problems for (3) is not developed even for the case of finite dimensions. In general, the standard Cauchy problem with conditions $\left.D_{t}^{i} u\right|_{t=0}=g_{i}\left(x^{\prime}\right), \quad i=0, \ldots, l-1$, for (3) has no classical solutions for an arbitrary right part $f(x)$.

The motive of our investigations is the wish to conceive the statement of initial and boundary value problems for the systems of partial differential equations with the Fredholm operator in the main part and also their applications. In this paper we show that we can get a reasonable statement of the initial problems for such systems by decomposing the space $E_{1}$ on the direct sum of subspaces in accordance with the Jordan structure ( [8], [10]) of the operator coefficients $B_{\alpha}$, and imposing conditions on projections of the solution. Here we suppose that $B$ is a closed Fredholm operator, $D(B) \subseteq D\left(B_{\alpha}\right) \forall \alpha$, and among the coefficients $B_{\alpha}$ there is an operator $A=B_{l_{1} 0 \ldots 0}, l_{1}<l$, with respect to which $B$ has the complete $A$-Jordan set 10 .

In Section 1 the sufficient conditions of existence of the unique solution of equation (3) with the initial conditions

$$
\begin{gathered}
\left.D_{t}^{i} u\right|_{t=0}=g_{i}\left(x^{\prime}\right), \quad i=0,1, \ldots, l_{1}-1, \\
\left.(I-P) D_{t}^{i} u\right|_{t=0}=g_{i}\left(x^{\prime}\right), \quad i=l_{1}, \ldots, l-1,
\end{gathered}
$$

are obtained, where $g_{i}\left(x^{\prime}\right)$ are analytical functions with values in $E_{1}, P g_{i}\left(x^{\prime}\right)=$ $0, \quad i=l_{1}, \ldots, l-1$. Here $P$ is the projector of $E_{1}$ onto corresponding $A$-root subspace. In Section 2 the left and right regularizators of singular operators in Banach spaces have been constructed. A method of fundamental operators for construction of the solution in the class of Schwarz distributions [6] is considered in Section 3. We hope that these investigations can be useful for considering of new applications [8], 9] of singular differential systems.

\section{Selection of Projection Operators and Reduction of the Initial Problem to the Kovalevskaya Form}

Suppose the following condition is satisfied:

Condition 1 [10]. The Fredholm operator $B$ has a complete A-Jordan set $\varphi_{i}^{(j)}$, $B^{*}$ has a complete $A^{*}$-Jordan set $\psi_{i}^{(j)}, \quad i=\overline{1, n}, j=\overline{1, p_{i}}$, and the systems 
$\gamma_{i}^{(j)} \equiv A^{*} \psi_{i}^{\left(p_{i}+1-j\right)}, z_{i}^{(j)} \equiv A \varphi_{i}^{\left(p_{i}+1-j\right)}, i=\overline{1, n}, j=\overline{1, p_{i}}$, corresponding to them are biorthogonal. Here $p_{i}$ are the lengths of the Jordan chains of the operator $B$.

Recall that condition 1 is satisfied if the operator $B+\lambda A$ is continuously invertible when $0<|\lambda|<\epsilon[10]$.

We introduce the projectors

$$
\begin{aligned}
& P=\sum_{i=1}^{n} \sum_{j=1}^{p_{i}}<., \gamma_{i}^{(j)}>\varphi_{i}^{(j)} \equiv(<., \Upsilon>\Phi), \\
& Q=\sum_{i=1}^{n} \sum_{j=1}^{p_{i}}<., \psi_{i}^{(j)}>z_{i}^{(j)} \equiv(<., \Psi>Z),
\end{aligned}
$$

generating the direct decompositions

$$
E_{1}=E_{1 k} \oplus E_{1 \infty-k}, E_{2}=E_{2 k} \oplus E_{2 \infty-k},
$$

where $k=p_{1}+\cdots+p_{n}$ is a root number. Then any solution of equation (3) can be represented in the form

$$
u(x)=\Gamma v(x)+(C(x), \Phi)
$$

where $\Gamma=\left(B+\sum_{i=1}^{n}<., \gamma_{i}^{(1)}>z_{i}^{(1)}\right)^{-1}$ is a bounded operator [10],

$$
\begin{gathered}
v \in E_{2 \infty-k}, C(x)=\left(C_{11}(x), \ldots, C_{1 p_{1}}(x), \ldots, C_{n 1}(x), \ldots, C_{n p_{n}}(x)\right)^{T}, \\
\Phi=\left(\varphi_{1}^{(1)}, \ldots, \varphi_{1}^{\left(p_{1}\right)}, \ldots, \varphi_{n}^{(1)}, \ldots, \varphi_{n}^{\left(p_{n}\right)}\right)^{T}
\end{gathered}
$$

where $T$ denotes transposition. The unknown functions $v(x): \Omega \subset R^{m+1} \rightarrow$ $E_{2 \infty-k}$ and $C(x): \Omega \subset R^{m+1} \rightarrow R^{k}$ due to initial conditions (4), (5)), satisfy the following conditions

$$
\begin{gathered}
\left.D_{t}^{i} v\right|_{t=0}=\left\{\begin{array}{l}
B(I-P) g_{i}\left(x^{\prime}\right), i=0, \ldots, l_{1}-1, \\
B g_{i}\left(x^{\prime}\right), i=l_{1}, \ldots, l-1,
\end{array}\right. \\
\left.D_{t}^{i} C\right|_{t=0}=\beta_{i}\left(x^{\prime}\right), i=0, \ldots, l_{1}-1 .
\end{gathered}
$$

Here $\beta_{i}\left(x^{\prime}\right)$ are coefficients of projections $P g_{i}\left(x^{\prime}\right), i=0, \ldots, l_{1}-1$.

Condition 2 The operator coefficients $B_{\alpha}$ in (3) satisfy at least one of five conditions on $D\left(B_{\alpha}\right)$ :

1. $B_{\alpha} P=Q B_{\alpha}$, i.e. $B_{\alpha}(P, Q)$-commute, briefly, $\alpha \in q_{0}$;

2. $B_{\alpha} P=0$, briefly $\alpha \in q_{1}$;

3. $Q B_{\alpha}=0$, briefly $\alpha \in q_{2}$;

4. $(I-Q) B_{\alpha}=0$, briefly $\alpha \in q_{3}$;

5. $B_{\alpha}(I-P)=0$, briefly $\alpha \in q_{4}$; 
We introduce the scalar product $(\Phi, C)=\sum_{i=1}^{n} \sum_{j=1}^{p_{i}} \varphi_{i}^{(j)} C_{i j}$. Then

$$
<B_{\alpha}(\Phi, C), \Psi>=\mathcal{A}^{\mathcal{T}}{ }_{\alpha} C
$$

where $\Psi=\left(\psi_{1}^{(1)}, \ldots, \psi_{1}^{\left(p_{1}\right)}, \ldots, \psi_{n}^{(1)}, \ldots, \psi_{n}^{\left(p_{n}\right)}\right)^{T}$. Due to condition 1 and lemma 3 [7] $\alpha \in q_{0}$, if and only if

$$
B_{\alpha}^{*} \Psi=\mathcal{A}_{\alpha}^{\mathcal{T}} \Upsilon, B_{\alpha} \Phi=\mathcal{A}_{\alpha} Z
$$

The operators $B \equiv B_{l 0 \ldots 0}, \quad A \equiv B_{l_{1} 0 \ldots 0}$ belong to the set $q_{0}$. Moreover the matrices of $(P, Q)$-commutability are symmetrical cell-diagonal matrices:

$$
\mathcal{A}_{l 0 \cdots 0}=\operatorname{diag}\left(B_{1}, \ldots, B_{n}\right), \mathcal{A}_{l_{1} 0 \cdots 0}=\operatorname{diag}\left(A_{1}, \ldots, A_{n}\right),
$$

where

$$
B_{i}=\left[\begin{array}{cccc}
0 & 0 & \ldots & 0 \\
0 & 0 & \ldots & 1 \\
\ldots & \ldots & \ldots & \ldots \\
0 & 1 & \ldots & 0
\end{array}\right], \quad A_{i}=\left[\begin{array}{ccc}
0 & \ldots & 1 \\
\ldots \ldots & \ldots \\
1 & \ldots & 0
\end{array}\right], \quad i=\overline{1, n}
$$

if $p_{i} \geq 2$ and $B_{i}=0, A_{i}=1$ if $p_{1}=1$.

$$
\mathcal{A}_{l 0 \cdots 0}=0, \quad \mathcal{A}_{l_{1} 0 \cdots 0}=E
$$

if $k=n$.

Note that due to the structure of projectors $P, Q$ the identity $\Gamma Q=P \Gamma$ holds. The spaces $E_{2 k}, E_{2 \infty-k}$ are invariant subspaces of the operator $\Gamma$. Taking into account that operator $\Gamma$ is a bounded one, $D(B) \subseteq D\left(B_{\alpha}\right)$ and $\overline{D\left(B_{\alpha}\right)}=E_{1}$, we obtain $B_{\alpha} \Gamma \in L\left(E_{1} \rightarrow E_{2}\right)$.

Thus by substituting (6) into (3) and projecting the result onto the subspace $E_{2 \infty-k}$, we obtain the equation

$$
D_{t}^{l} v+(I-Q) \sum^{\prime} B_{\alpha} \Gamma D^{\alpha} v=(I-Q)\left(f-\sum^{\prime \prime} B_{\alpha}\left(D^{\alpha} C, \Phi\right)\right)
$$

where

$$
\sum^{\prime}=\sum_{|\alpha| \leq l, \alpha \in\left(q_{0}, q_{1}, q_{2}\right) \backslash(l 0 \ldots 0)}, \sum^{\prime \prime}=\sum_{|\alpha| \leq l, \alpha \in\left(q_{2}, q_{4}\right)}
$$

with condition (17). Similarly, we project (3), where $u$ is defined by ([6), onto the subspace $E_{2 k}$ and obtain the system

$$
\sum_{|\alpha| \leq l, \alpha \in\left(q_{0}, q_{3}, q_{4}\right)} M_{\alpha} D^{\alpha} C=b(x, v)
$$

with initial condition (8). Thus the initial problem (3), (41), (15) is reduced to problems (11), (7) and (12), (8). 
In system (12)

$$
M_{\alpha}=\left\|<B_{\alpha} \varphi_{l}^{(s)}, \psi_{i}^{(j)}>\right\|, i, l=1, \ldots n, j=1, \ldots, p_{i}, \quad s=1, \ldots, p_{l},
$$

are matrices of the dimension $k \times k, b(x, v)$ is the vector of projection coefficients

$$
Q\left(f-\sum_{|\alpha| \leq l, \alpha \in\left(q_{1}, q_{3}\right)} B_{\alpha} \Gamma D^{\alpha} v\right) .
$$

Recall that if $\alpha \in q_{0}$, then $M_{\alpha}=\mathcal{A}_{\alpha}^{T}$. Thus, for $k=n$ according to (10) it follows that

$$
M_{l 0 \ldots 0}=0, M_{l_{1} 0 \ldots 0}=E,
$$

and for $k>n$ the matrices $M_{l 0 \ldots 0}, M_{l_{1} 0 \ldots 0}$ are defined from (91).

Theorem 1. Suppose conditions 1 and 2 are satisfied, the function $f(x)$ is an analytical on $x^{\prime}$ and sufficiently smooth on $t$. Suppose

1. $\left(q_{2}, q_{4}\right) \subset q_{0}$ or $\left(q_{1}, q_{3}\right) \subset q_{0}$;

2. $Q B_{\alpha} P=0$ for all $\alpha \in\left(q_{0}, q_{3}, q_{4}\right) \backslash(l 0 \ldots 0),\left(l_{1} 0 \ldots 0\right)$.

Then the problem (3), (4), (5) has a unique classical solution (6).

Proof. Note that for $\alpha \in q_{0}$, and for any $C$ the equality $(I-Q) B_{\alpha}\left(D^{\alpha} C, \Phi\right)=0$ holds and $Q B_{\alpha} \Gamma v=0$, where $Q v=0$. Thus, according to condition 1 of this theorem the right-hand side of (11) is independent on the vector-function $C(x)$, or the right-hand side of (12) is independent on $\mathrm{v}(\mathrm{x})$. The equation (11) is solvable with respect to $D_{t}^{l} v$, i.e. has the Kovalevskaya form with the bounded operator coefficients. Due to condition 2 of this theorem the system (12) takes the following form

$$
M_{l 0 \ldots 0} D_{t}^{l} C+M_{l_{1} 0 \ldots 0} D_{t}^{l_{1}} C=b(x, v) .
$$

If $k=n$, then $M_{l 0 \ldots 0}=0, \quad M_{l_{1} 0 \ldots 0}=E$ and system (13) has the order $l_{1}$. If $k>n$ then system (13) is split on $n$ independent subsystems:

$$
\frac{\partial^{l_{1}}}{\partial t^{l_{1}}} C_{i p_{i}}=b_{i p_{i}}(x, v), \quad \frac{\partial^{l_{1}}}{\partial t^{l_{1}}} C_{i p_{i}-k}+\frac{\partial^{l}}{\partial t^{l}} C_{i p_{i}-k+1}=b_{i p_{i}-k}(x, v),
$$

where $i=\overline{1, n}, \quad k=\overline{1, p_{i}-1}$. Each subsystem (14) is regular, since it is a recurrent sequence of differential equations of order $l_{1}$. Thus systems (11), (12) with boundary conditions (17), (18) have the Kovalevskaya form and therefore have the unique solution. Taking $v$ and $C$ from the regular systems (11), (12) and substituting them into (6), we obtain the needed solution.

Remark 1. Let the operators $B_{\alpha}$ in condition 2 depend on $x$ for $\alpha \neq(l 0 \ldots 0)$, $\left(l_{1} 0 \ldots 0\right)$. Then the coefficients in the systems (11), (12) also depend on $x$. If these coefficients are analytical on $x^{\prime}$ and sufficiently smooth on $t$, then theorem 1 is valid. Required smoothness on $t$ for these coefficients and $f(x)$ is defined by maximum length of $A$-Jordan chains of operator $B$. 


\section{The Left and Right Regularizators of Singular Operators in Banach Spaces}

Let $A$ and $B$ be constant linear operators from $E_{1}$ to $E_{2}$, where $E_{1}$ and $E_{2}$ are Banach spaces, $x(t)$ is an abstract function, $t \in R_{n}$ with the values in $E_{1}\left(E_{2}\right)$. The set of such functions we denote by $X_{t}\left(Y_{t}\right)$. We introduce the operator $L_{t}$, defined on $X_{t}$ and $Y_{t}$ and which is commutable with operators $B, A$. The examples of such operator $L_{t}$ are differential and integral operators, difference operators and their combinations. Note that if operators are solved according to higher order derivatives, then they usually generate correct initial and boundary value problems. In other cases, when operators are unsolved according to higher order derivatives, we get the singular problems (see sec.1).

Let us consider the operator $L_{t} B-A$, which acts from $X_{t}$ to $Y_{t}$, where $B, A$ are closed linear operators from $E_{1}$ to $E_{2}$ with the dense domains, and $D(B) \subseteq D(A)$. If $B$ is invertable, then the operator $L_{t} B-A$ can be reduced to regular operator by multiplication on $B^{-1}$. If $B$ is uninvertable, then $L_{t} B-A$ is called the singular operator. Let operator $B$ in $L_{t} B-A$ be Fredholm and $\operatorname{dim} N(B)=n \geq 1$. If $\lambda=0$ is an isolated singular point of the operatorfunction $B-\lambda A$, then the operators $L_{t} B-A, B L_{t}-A$ admit some regularization. For explicit construction of regularizations we use the Schmidt pseudo resolvent $\Gamma=\hat{B}^{-1}$, where $\hat{B}=B+\sum_{i=1}^{n}<., A^{*} \psi_{i}^{\left(p_{i}\right)}>A \phi_{i}^{\left(p_{i}\right)}$. On the base of condition 1 ( sec. 1) and using the equalities $\phi_{i}^{(j)}=\Gamma A \phi_{i}^{(j-1)}, \quad \psi_{i}^{(j)}=\Gamma^{*} A^{*} \psi_{i}^{(j-1)}, \quad j=$ $2, \ldots, p_{i}, \quad i=1, \ldots, n$ it is easy to check the following equalities

$$
\begin{aligned}
& \left(\Gamma-\sum_{i=1}^{n} \sum_{j=1}^{p_{i}} L_{t}^{j}<., \psi_{i}^{\left(p_{i}+1-j\right)}>\phi_{i}\right)\left(L_{t} B-A\right)=L_{t}-\Gamma A \\
& \left(L_{t} B-A\right)\left(\Gamma-\sum_{i=1}^{n} \sum_{j=1}^{p_{i}} L_{t}^{p_{i}+1-j}<., \psi_{i}>\phi_{i}^{(j)}\right)=L_{t}-A \Gamma .
\end{aligned}
$$

Thus we have the following

Theorem 2. Suppose condition 1 in section 1 is satisfied. Then

$$
\left(\Gamma-\sum_{i=1}^{n} \sum_{j=1}^{p_{i}} L_{t}^{j}<., \psi_{i}^{\left(p_{i}+1-j\right)}>\phi_{i}\right)
$$

and

$$
\Gamma-\sum_{i=1}^{n} \sum_{j=1}^{p_{i}} L_{t}^{p_{i}+1-j}<., \psi_{i}>\phi_{i}^{(j)}
$$

are the left and right regularizators of $L_{t} B-A$, respectively.

Note that these results are applicable for the investigation of singular differential-operator equations with the Fredholm operator in the main part (see [1]). 


\section{Fundamental Operator-Functions of Singular Partial Differential and Differential-Difference Operators in Banach Spaces}

Since the standard Cauchy problem for equation (3) with the Fredholm operator $B_{l 0 \ldots 0}$ in general has no classical solution, then it will be interesting to extend the notion of solution and to seek generalized solution in a distribution space 6 .

The most interesting is the construction of the fundamental operator functions for the singular differential operators in Banach spaces which help to obtain the generalized solutions in closed forms.

Here we construct the fundamental operator functions for the following mappings

$$
B \frac{\partial^{2 N} u}{\partial x^{N} \partial y^{N}}-A u, \quad B \frac{\partial u}{\partial t}-A(u(t, x-\mu)-u(t, x)),
$$

where $B$ is a Fredholm operator.

The basic information on generalized functions in Banach spaces, their properties and operations can be found in [3], 8].

Theorem 3. Suppose that condition 1 is satisfied. Then the mapping $B \delta^{\prime}(x) \delta^{\prime}(y)-A \delta(x) \delta(y)$ in the space $K^{\prime}\left(E_{2}\right)$ has the fundamental operator function of the form

$$
\begin{aligned}
\mathcal{E}_{1}(x, y)= & \Gamma \mathcal{U}_{1}(A \Gamma)(x, y)[I-Q] \theta(x, y) \\
& -\sum_{i=1}^{n} \sum_{k=0}^{p_{i}-1}\left\{\sum_{j=1}^{p_{i}-k}\left\langle\cdot, \psi_{i}^{(j)}\right\rangle \varphi_{i}^{\left(p_{i}-k+1-j\right)}\right\} \delta^{(k)}(x) \cdot \delta^{(k)}(y),
\end{aligned}
$$

where

$$
\mathcal{U}_{1}(A \Gamma)(x, y)=\sum_{i=0}^{\infty}(A \Gamma)^{i} \cdot \frac{x^{i}}{i !} \cdot \frac{y^{i}}{i !}
$$

Proof. In accordance with the definition it is necessary to check up a validity of equality

$$
\left(B \delta^{\prime}(x) \cdot \delta^{\prime}(y)-A \delta(x) \cdot \delta(y)\right) * \mathcal{E}_{1}(x, y) * u(x, y)=u(x, y)
$$

on the basic space $K\left(E_{2}^{*}\right)$. Let us substitute the expression for $\mathcal{E}_{1}(x, y)$ into the left-hand side of this equality

$$
\begin{aligned}
& \left(B \delta^{\prime}(x) \cdot \delta^{\prime}(y)-A \delta(x) \cdot \delta(y)\right) * \mathcal{E}_{1}(x, y) * u(x, y) \\
& =\left(B \Gamma A \Gamma \mathcal{U}_{1}(A \Gamma)(x, y)[I-Q] \theta(x, y)+B \Gamma[I-Q] \delta(x) \cdot \delta(y)\right. \\
& \quad-\sum_{i=1}^{n} \sum_{k=1}^{p_{i}-1}\left\{\sum_{j=1}^{p_{i}-k}\left\langle\cdot, \psi_{i}^{(j)}\right\rangle\left(B \varphi_{i}^{\left(p_{i}-k+2-j\right)}-A \varphi_{i}^{\left(p_{i}-k+1-j\right)}\right)\right\} \delta^{(k)}(x) \cdot \delta^{(k)}(y)
\end{aligned}
$$




$$
\left.-A \Gamma \mathcal{U}_{1}(A \Gamma)(x, y)[I-Q] \theta(x, y)+Q \delta(x) \cdot \delta(y)\right) * u(x, y)
$$

Since $B \Gamma=I-\sum_{i=1}^{n}\left\langle\cdot, \psi_{i}^{(1)}\right\rangle z_{i}, z_{i}=A \varphi_{i}^{\left(p_{i}\right)}, B \varphi_{i}^{(j)}=A \varphi_{i}^{(j-1)}$, then

$$
\sum_{i=1}^{n}\left\langle\cdot, \psi_{i}^{(1)}\right\rangle z_{i}[I-Q]=0, \sum_{i=1}^{n}\left\langle\cdot, \psi_{i}^{(1)}\right\rangle z_{i} A \Gamma \mathcal{U}_{1}(A \Gamma)(x, y)[I-Q]=0
$$

and

$$
\left(B \delta^{\prime}(x) \cdot \delta^{\prime}(y)-A \delta(x) \cdot \delta(y)\right) * \mathcal{E}_{1}(x, y) * u(x, y)=I \delta(x) \cdot \delta(y) * u(x, y)=u(x, y)
$$

The following theorem can be proved similarly.

Theorem 4. Suppose condition 1 is satisfied, then the mapping $B \delta^{(N)}(x)$. $\left.\delta^{(N)}(y)-A \delta(x) \cdot \delta(y)\right)$ in the space $K^{\prime}\left(E_{2}\right)$ has the fundamental operator function of the form

$$
\begin{aligned}
\mathcal{E}_{N}(x, y)= & \Gamma \mathcal{U}_{N}(A \Gamma)(x, y)[I-Q] \theta(x, y) \\
& -\sum_{i=1}^{n} \sum_{k=0}^{p_{i}-1}\left\{\sum_{j=1}^{p_{i}-k}\left\langle\cdot, \psi_{i}^{(j)}\right\rangle \varphi_{i}^{\left(p_{i}-k+1-j\right)}\right\} \delta^{(k \cdot N)}(x) \cdot \delta^{(k \cdot N)}(y),
\end{aligned}
$$

where

$$
\mathcal{U}_{N}(A \Gamma)(x, y)=\sum_{i=1}^{\infty}(A \Gamma)^{i-1} \cdot \frac{x^{i \cdot N-1}}{(i \cdot N-1) !} \cdot \frac{y^{i \cdot N-1}}{(i \cdot N-1) !}
$$

As a corollary of theorem 3 we obtain

Corollary 1. Suppose condition 1 is satisfied, the function $f(x, y) \in C\left(R_{+}^{2}\right)$ takes value in $E_{2}$. Then the boundary value problem

$$
B \frac{\partial^{2} u}{\partial x \partial y}=A u+f(x, y),\left.\quad u\right|_{x=0}=\alpha(y),\left.u\right|_{y=0}=\beta(x),
$$

$u(x, y) \in C^{2}\left(R_{+}^{2}\right), \alpha(x), \beta(x) \in C^{1}\left(R_{+}^{1}\right), \alpha(0)=\beta(0)$, has a generalized solution of the form

$$
\begin{aligned}
u= & \mathcal{E}_{1}(x, y) *\left(f(x, y) \theta(x, y)+B \alpha^{\prime}(y) \delta(x) \cdot \theta(y)\right. \\
& \left.+B \beta^{\prime}(x) \theta(x) \cdot \delta(y)+B \alpha(0) \delta(x) \cdot \delta(y)\right)
\end{aligned}
$$

If additionally the singular components of the generalized solutions are equal to zero then, firstly, generalized solutions coincide with continuous (classical) solutions, and, secondly, we can define a set of the boundary values $\alpha(y)$ and $\beta(x)$ and right sides $f(x, y)$, for which such problems are solvable in the class of functions $C^{2}\left(R_{+}^{2}\right)$. 
Remark 2. The following boundary value problem can be investigated similarly $B \frac{\partial^{2 N} u}{\partial x^{N} \partial y^{N}}=A u+f(x, y),\left.\frac{\partial^{i} u}{\partial x^{i}}\right|_{x=0}=\alpha_{i}(y),\left.\frac{\partial^{i} u}{\partial y^{i}} u\right|_{y=0}=\beta_{i}(x), i=0, \ldots, N-1$.

Theorem 5. Suppose that condition 1 is satisfied with $p_{1}=p_{2}=\ldots p_{n}=1$. Then the mapping $B \delta^{\prime}(t) \cdot \delta(x)-A \delta(t) \cdot(\delta(x-\mu)-\delta(x))$ in the space $K^{\prime}\left(E_{2}\right)$ has the fundamental operator function of the form

$\mathcal{E}(t, x)=\sum_{k=0}^{\infty} \Gamma e^{-A \Gamma t} \frac{(A \Gamma t)^{k}}{k !} \theta(t) \cdot \delta(x-\mu) *\left\{I \delta(t) \cdot \delta(x)+Q \delta^{\prime}(t) \cdot \sum_{j=0}^{\infty} \delta(x-j \mu)\right\}$.

Proof. In accordance with the definition it is necessary to check up a validity of equality

$$
\left(B \delta^{\prime}(t) \cdot \delta(x)-A \delta(t) \cdot(\delta(x-\mu)-\delta(x))\right) * \mathcal{E}(t, x) * u(t, x)=u(t, x)
$$

on the basic space $K\left(E_{2}^{*}\right)$. Let us substitute the expression for $\mathcal{E}(t, x)$ into the left-hand side of this equality

$$
\begin{aligned}
\left(B \delta^{\prime}(t) \cdot \delta(x)-A \delta(t) \cdot(\delta(x-\mu)-\delta(x))\right) * \mathcal{E}(t, x) * u(t, x) & \\
= & {[I \delta(t) \cdot \delta(x)+F(t, x)] * u(t, x), }
\end{aligned}
$$

where

$$
\begin{aligned}
F(t, x)= & -\sum_{k=0}^{\infty} Q\left(e^{-t} \frac{t^{k}}{k !} \theta(t)\right)^{\prime} \cdot \delta(x-k \mu)+[I \delta(t) \cdot \delta(x) \\
& \left.\left.-\sum_{k=0}^{\infty} Q\left(e^{-t} \frac{t^{k}}{k !} \theta(t)\right)^{\prime} \cdot \delta(x-k \mu)\right)\right] * Q \delta^{\prime}(t) \cdot \sum_{j=0}^{\infty} \delta(x-j \mu)=0 .
\end{aligned}
$$

Remark 3. In theorem $4 x$ can be vector, moreover theorem 5 keeps its validity if $p_{i} \geq 1, i=1, \ldots, n$, and differential operator can be changed on differentialdifference operator

$$
B \delta^{(N)}(t) \cdot \delta(x)-A \delta(t) \cdot(\delta(x-\mu)-\delta(x)) .
$$

Corollary 2. Suppose condition of theorem 5 is satisfied, the function $f(t, x) \in$ $B U C\left(R^{1}\right)$ [4], $\forall t \geq 0$, takes value in $E_{2}$. Then the Cauchy problem for differential-difference equation

$$
B \frac{\partial u}{\partial t}=A(u(t, x-\mu)-u(t, x))+f(t, x),\left.\quad u\right|_{t=0}=u_{0}(x),
$$

where $u_{0}(x) \in B U C\left(R^{1}\right)$, has a generalized solution of the form

$$
u=\mathcal{E}(t, x) *\left(f(t, x) \theta(t)+B u_{0}(x) \delta(t)\right) .
$$




\section{References}

1. Chistyakov, V.F.: Algebro-differential equations with finite-dimensional kernel. Nauka, Novosibirsk (1996)

2. Korpusov, M.O., Pletnev,Y.D., Sveshnikov, A.G.: On quasi-steddy process in the conducting medium without dispersion. Comput. Math. and Math. Phys. Vol.40. 8 (2000) 1237-1249

3. Falaleev, M.V.: Fundamental operator functions of singular differential operators in Banach spaces. Sib. Math. J. 41 (2000) 960-973

4. Goldstein, J.A.: Semigroups of Linear Operators and Applications. Oxford University Press, Inc., New York (1985)

5. Petrovsky, I.: Uber das Caushysche problem fur system von partiellen Differentialgleichunden. Math. Sbornic Vol.2. 5 (1937) 815-870

6. Schwartz, L.: Theorie des distributions. I-II Paris (1950-1951)

7. Sidorov, N.A., Blagodatskaya, E.B.: Differential Equations with the Fredholm Operator in the Leading Differential Expression. Soviet Math. Dokl. Vol.44. 1 (1992) 302-305

8. Sidorov N., Loginov B., Sinitsyn A., Falaleev M.: Lyapunov-Schmidt Methods in Nonlinear Analysis and Applications. Book Series: Mathematics and Its Applications: 550 Kluwer Academic Publishers (2002)

9. Sviridyuk, G.A.: On the General Theory of Semigroup of Operators. Uspekhi Mat. Nauk. 49 (1994) 47-74

10. Vainberg, M.M., Trenogin, V.A.: The Theory of Branching of Solutions of Nonlinear Equations. Wolters-Noordhoff, Groningen (1974) 